\title{
Biological activities of fusarochromanone: a potent anti-cancer agent
}

\author{
Elahe Mahdavian ${ }^{1 *}$, Phillip Palyok', Steven Adelmund ${ }^{1}$, Tara Williams-Hart ${ }^{2}$, Brian D Furmanski ${ }^{3}$, Yoon-Jee Kim",

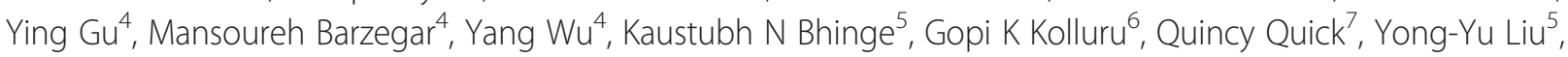 \\ Christopher G Kevil ${ }^{6}$, Brian A Salvatore', Shile Huang ${ }^{4}$ and John L Clifford ${ }^{8}$
}

\begin{abstract}
Background: Fusarochromanone (FC101) is a small molecule fungal metabolite with a host of interesting biological functions, including very potent anti-angiogenic and direct anti-cancer activity.

Results: Herein, we report that FC101 exhibits very potent in-vitro growth inhibitory effects ( $\mathrm{I}_{50}$ ranging from 10nM-2.5 $\mu \mathrm{M}$ ) against HaCat (pre-malignant skin), P9-WT (malignant skin), MCF-7 (low malignant breast), MDA-231 (malignant breast), SV-HUC (premalignant bladder), UM-UC14 (malignant bladder), and PC3 (malignant prostate) in a time-course and dose-dependent manner, with the UM-UC14 cells being the most sensitive. FC101 induces apoptosis and an increase in proportion of cells in the sub-G1 phase in both HaCat and P9-WT cell lines as evidenced by cell cycle profile analysis. In a mouse xenograft SCC tumor model, FC101 was well tolerated, non-toxic, and achieved a 30\% reduction in tumor size at a dose of $8 \mathrm{mg} / \mathrm{kg} / \mathrm{day}$. FC101 is also a potent anti-angiogenenic agent. At nanomolar doses, FC101 inhibits the vascular endothelial growth factor-A (VEGF-A)-mediated proliferation of endothelial cells.

Conclusions: Our data presented here indicates that FC101 is an excellent lead candidate for a small molecule anti-cancer agent that simultaneously affects angiogenesis signaling, cancer signal transduction, and apoptosis. Further understanding of the underlying FC101's molecular mechanism may lead to the design of novel targeted and selective therapeutics, both of which are pursued targets in cancer drug discovery.
\end{abstract}

Keywords: Pro-apoptotic, Anti-cancer, Anti-angiogenic, Small bioactive molecule, Fusarochromanone

\section{Background}

Fusarochromanone (FC101) is a mycotoxin that is produced by the symbiotic fungus, Fusarium equiseti, found on decaying cereal plants from northern latitudes. FC101 was originally discovered to cause avian tibial dyschondroplasia (ATD) in broiler chickens [1,2]. It also reduced hatchability in fertile eggs when birds were fed diets containing Fusarium-infected feed $[1,3]$. This toxin is also suspected of being involved in etiopathogenesis of KashinBeck disease in children from northern China, Siberia, the former USSR, and Korea [4]. Lee et al. first isolated this molecule and determined its structure (without stereochemistry) via NMR and mass spectrometry [5]. FC101 is

\footnotetext{
* Correspondence: emahdavi@lsus.edu

'Department of Chemistry and Physics, LSU-Shreveport, One University Place, Shreveport, LA 71115, USA

Full list of author information is available at the end of the article
}

distinguished from other known chromanone natural products by the unique arrangement of the alternating $\beta$-keto-amine functionality along its upper face and two geminal methyl groups at $C(2)$ (Figure 1). Using X-Ray crystallography, the absolute stereochemistry at $C\left(3^{\prime}\right)$ was defined as 3'-R, according to the Cahn-Inglold-Prelog convention (Smith, unpublished observations). The amine group at $\mathrm{C}\left(3^{\prime}\right)$ on the side-chain is in fact important to the molecule's biological activity, since its acetylation drastically reduces the activity [6]. Wuthier reported that FC101's inhibition of calcification in the legs of baby chickens was likely the result of its anti-angiogenic activity [7]. This effect is associated with a thickening of the cartilage on the tibial growth plate and a failure of this tissue to vascularize and calcify.

In addition to its anti-angiogenic properties in chickens, FC101 is also a potent anti-angiogenic agent in 


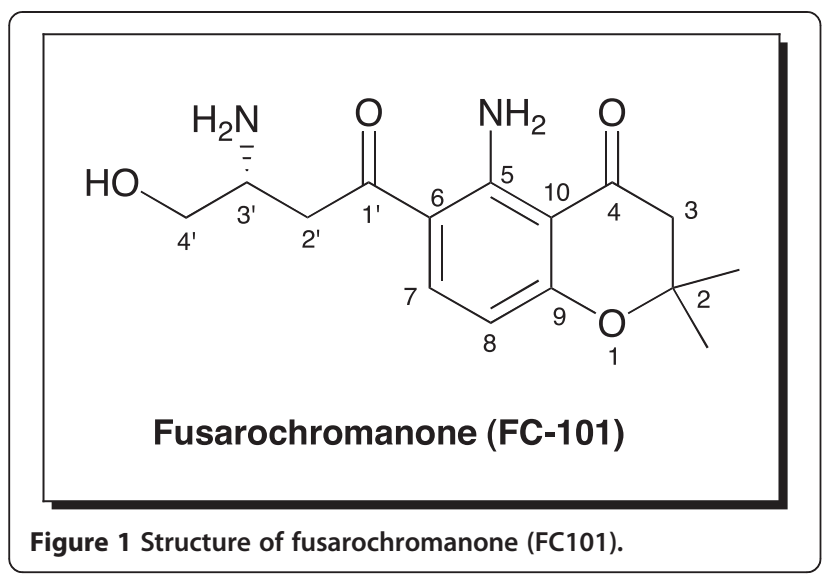

humans. FC101 has an $\mathrm{IC}_{50}$ value of $50 n M$ against human microvascular endothelial cells (NCI, unpublished). Those initial cell studies were done in the absence of any angiogenic factors.

It was later discovered that FC101 also acts directly on cancer cells. A 60-cell-line drug screening assay performed by the National Cancer Institute (NCI) revealed that FC101 inhibited the proliferation of 35 of 58 human cancer cell lines with $\mathrm{IC}_{50}$ 's of less than $100 \mathrm{nM}$ [NCI, unpublished]. The most sensitive cell lines were human melanoma, small cell lung carcinoma, and colon adenocarcinoma, with $\mathrm{IC}_{50}$ values all below $10 \mathrm{nM}$. The NCI also conducted a COMPARE screen on FC101. This screen applies algorithms that are used to assess the mode of action of a test compound. In this screen, FC101 inhibition was studied in the same 60 cell-line drug screening assay as mentioned above. These patterns were then compared to those found in a library of over 50,000 compounds. The data generated from the COMPARE test is represented as a Pearson correlation coefficient. Correlations greater the 0.8 indicate that the test molecule inhibits cellular growth in a similar manner to a compound found in the NCI database. Correlations below 0.6 are thought to have minimal, if not altogether different modes of action. The NCI COMPARE correlation factor for FC101 was 0.475 , indicating that FC101 is unique in its mode of action [NCI, unpublished].

Another unique attribute of FC101 is its intrinsic fluorescence, with a maximum excitation at $385 \mathrm{~nm}$ and emission at $457 \mathrm{~nm}$. A recent study utilized this intrinsic fluorescence to investigate the kinetics and uptake of FC101 by tumorigenic cells vs. normal cells in a rodent model analyzed by confocal microscopy [8]. This study reported an increased uptake of FC101 and growth inhibition in tumorgenic B16 melanoma and MCF-7 breast adenocarcinoma cells, as compared to the normal cardiac fibroblast cells. This group also reported experimental and in-silico values for a series of physiochemical properties (LogP, LogD, polar surface area, hydrogen bonding, molecular flexibility) that contribute to the bioavailability of FC101. They concluded that FC101 shows very good cell permeability and intestinal absorption, meeting the criteria for therapeutic drugs that were established by Lipinski et al. [9-11].

In this study we also tested the hypothesis that FC101's molecular targets (i.e., interacting biomolecules) could be regulators of angiogenesis, cancer signal transduction, cell proliferation, and/or programmed cell death (apoptosis). The detailed effects of FC101 on these processes were determined for a panel of human tumor cell types (skin squamous cell carcinoma [SCC], breast, prostate, and bladder). In addition, we tested the tumor suppressive effect of FC101 in vivo, in a mouse tumorigenicity model, against human skin SCC cells. SCC is the most aggressive form of non-melanoma skin cancer.

\section{Results}

It was previously reported in a mouse xenograft model for melanoma that FC101 induced expression of active caspase-3 [12]. Furthermore, our preliminary studies in yeast have shown that Ycal is involved in the response to FC101-treatment (Williams-Hart, unpublished). Yca1 is a caspase-like protein that has been shown to be important for executing apoptotic-like responses in yeast [13]. This finding led to the hypothesis that the tumor suppressive effects of FC101 may result from changes in the regulation of apoptotic signaling. In order to determine whether FC101 can induce cell death in culture, a preliminary indicator of apoptosis, several cell lines derived from different human tumor types were treated with a range of doses of FC101 over a course of four days. The MTT cell viability assay was used to obtain an indirect measure of cell number. All tumor cell types were sensitive to FC101 in a dose dependent manner, and in the case of the breast and bladder cancer cell lines, the more malignant cells were more sensitive to FC101 than the less malignant ones (Figure 2, compare $100 \mathrm{nM}$ curves in panel $\mathrm{C}$ to $\mathrm{D}$, and $\mathrm{E}$ to $\mathrm{F}$ ).

These results are more clearly indicated in Figure $2 \mathrm{H}$, where the percent growth inhibition for all 7 cell lines is shown for the day 4 time point (color of lines in graphs in Figure 2A-G corresponds to color of bars in Figure 2H). The UM-UC14 cells were the most sensitive of all of the cell lines; the 100nM dose drastically diminished cell number compared to other cells lines. It should be noted that this was the fastest growing cell line of the group tested (Figure 2F, compare the y-axis scale to the other graphs).

Since the MTT assay only measures cell metabolism/ mitochondrial respiration, which is an indirect measure of cell number, it cannot readily determine whether reduced cell number is caused by suppression of proliferation, or the induction of apoptosis. In order to determine which 

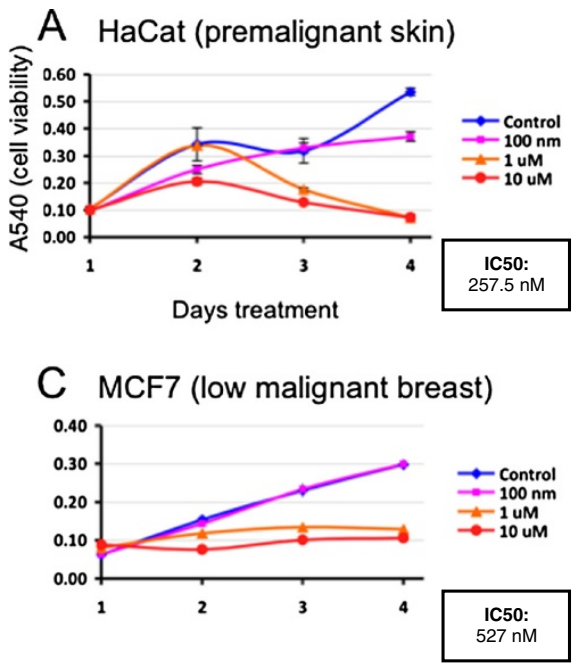

\section{E SV-HUC (premalignant bladder)}
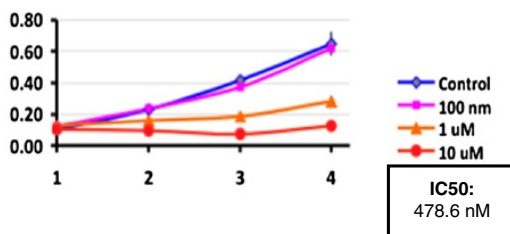

\section{G PC3 (malignant prostate)}

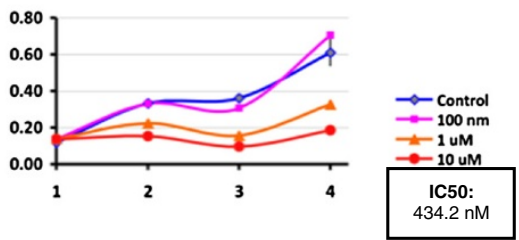

B SRB12-p9 (malignant skin)

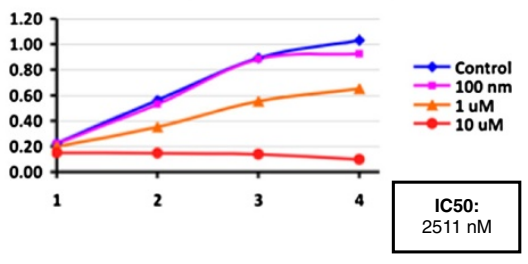

D MDA-MB231 (malignant breast)

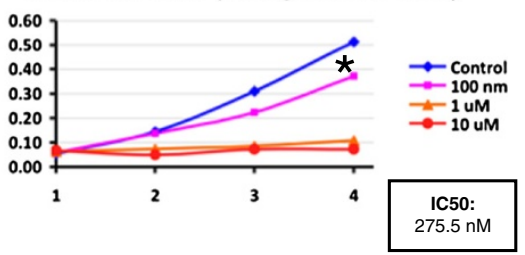

F

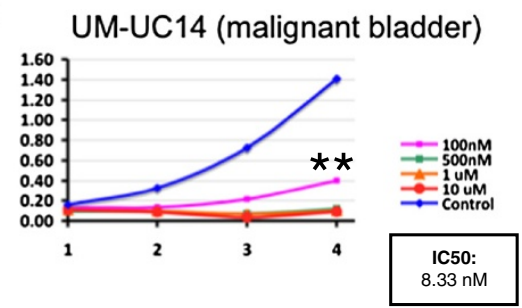

$\mathrm{H}$

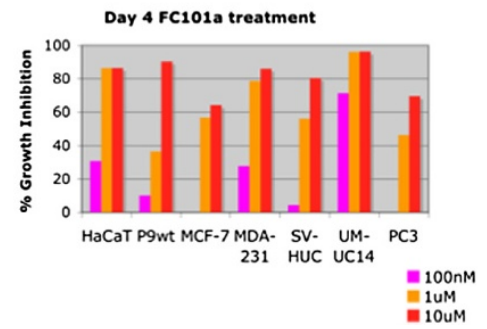

Figure 2 Comparison of the dose-response and time-response impact of FC101 treatment on cell viability of seven cell lines. A-G: A540 is the absorbance at $540 \mathrm{~nm}$, which positively correlates with cell number. Error bars are shown for triplicate cultures. The graphs are representative of at least three experiments having similar results. $\mathbf{H}$ : The percent growth inhibition at day 4, calculated as (A540C-A540T/A540)x100, where A540C is the control value and A540T is the treated value. The graph lines in A-G are color matched to the bars in $\mathbf{H}$.

process FC101 predominantly affects, we measured its effect on the cell cycle distribution. The HaCat premalignant keratinocyte and P9 WT skin SCC cell lines were chosen for this analysis, because these cell lines have been extensively studied in this context with other apoptosisinducing drugs.

Cells were treated for 24 and 48 hours with FC101 $(10 \mu \mathrm{M})$. These time points were chosen because marked effects of FC101 on viability were first detected after 48 hours of treatment (Figure 2A and B, compare 1 and 2 day time points). As with the MTT results for both cell lines, there was little effect of FC101 treatment after 24 hours (Figure 3, upper row, FC101 panels). However, after 48 hours there were fewer attached cells in both cell lines, with a stronger effect visible for the P9 WT cells
(Figure 3, lower panels). The presence of many rounded cells detaching from the plate is an indicator of cell death, possibly by apoptosis. In order to verify whether FC101 did indeed induce apoptosis, and whether there were any changes in the proliferative behavior of the cells, FACS analysis was performed for the same dose and time points of treatment. Treatment with FC101 for 24 hours caused an increase in percentage of cells in the $2 \mathrm{~N}$ DNA content peak, indicating cells in the G2 and M phases of the cell cycle for both cell lines (Figure 4, compare FC101 panels to controls in top row). The G2 phase is the point at which the DNA has been fully replicated, but prior to mitosis. This effect was accompanied by a modest increase in the percentage of particles that were smaller in size than G1 cells (the sub-G1 fraction) for both cell lines, 


\section{HaCat}
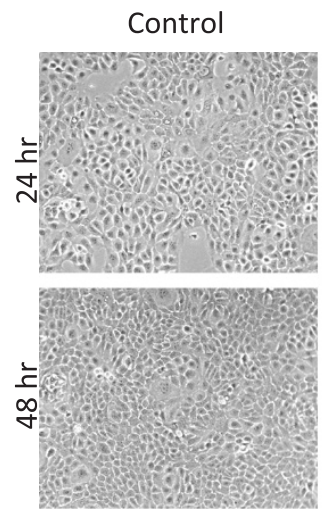

FC101a
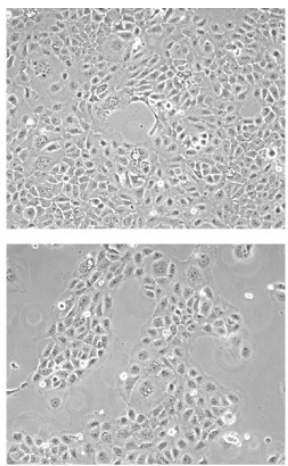

P9 WT
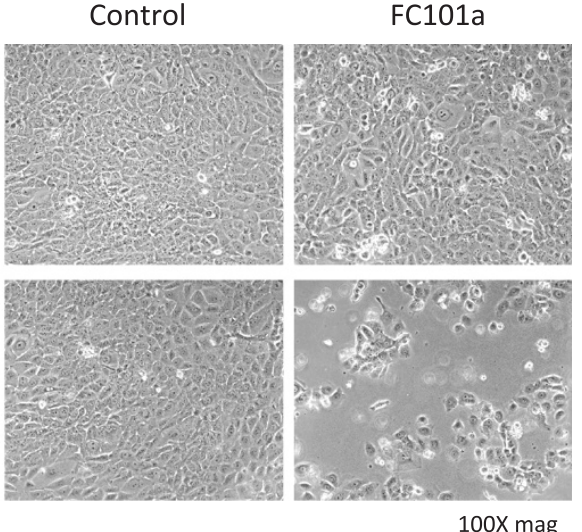

Figure 3 Cells were plated at a density of $1.5 \times 10^{6} \mathrm{cells} / 10 \mathrm{~cm}$ plate and treated the following day with $10 \mu \mathrm{M}$ FC101. Cells were photographed on a phase contrast microscope at $100 \mathrm{X}$ magnification.

indicating a modest increase in apoptosis with treatment. An enhanced fraction of cells in the $2 \mathrm{~N}$ peak was still observed after 48 hours of treatment, but the sub-G1 fraction was markedly increased compared to controls (Figure 4, lower panels). The increase in the percentage of cells in the $2 \mathrm{~N}$ peak was greater at both time points for the HaCaT cells compared to SRB12-p9 cells. However, the induction of apoptosis, as measured by the size of the sub-G1 fraction, was greater for the SRB12-p9 cells.

Programmed cell death can proceed through either caspase-dependent or independent routes [14]. To determine the mechanism of FC101-induction of apoptosis, MDA-MB-231 breast cancer cells were exposed to FC101 $(0-1 \mu \mathrm{M})$ for $24 \mathrm{~h}$, followed by western blotting

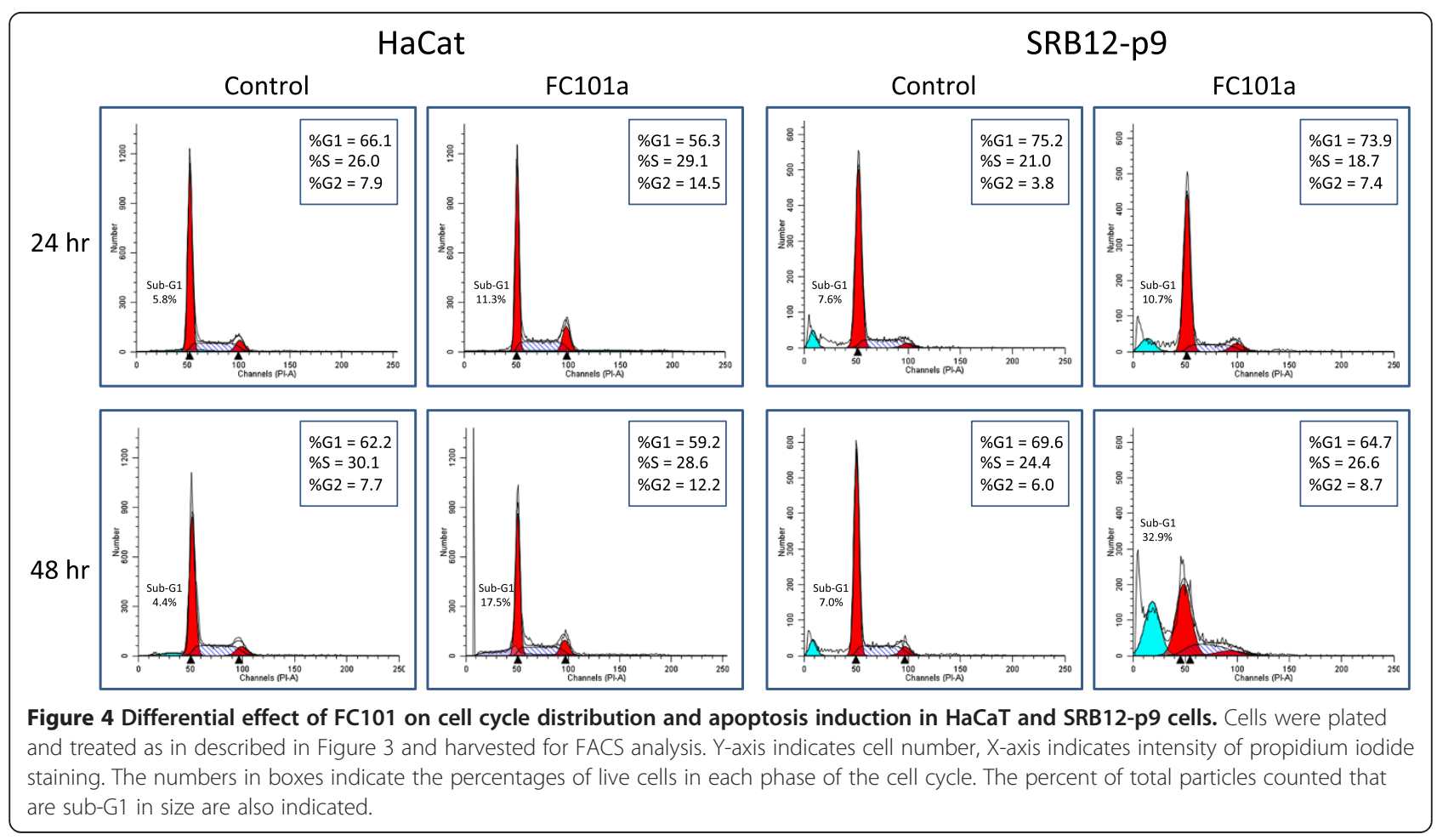


analysis to probe the differential expression and activities of the pro-apoptotic, anti-apoptotic, and caspase proteins involved in apoptosis.

FC101 induces the cleavage of both caspase- 3 and PARP, a well-known substrate for activated caspases (Figure 5). This indicates that FC101 activates the caspase signaling cascade. However, FC101 does not affect the expression of the anti-apoptotic proteins, Bcl-2, Bcl$\mathrm{XL}$, Mcl-1, or the pro-apoptotic proteins BAD, BAK, BAX (Figure 6). Thus, although FC101 activates apoptosis in a caspase-dependent manner, this activity does not involve a mitochondrial-mediated intrinsic mechanism. Furthermore, treatment with FC101 increases the activity of caspase-8, thus substantiating that FC101 initiates apoptotic cascades through a death receptor mediated extrinsic mechanism involving caspase- 8 activation (Figure 5).

Mitogen Activated Protein Kinases (MAPKs) are also involved in programmed cell death [15] and are frequently implicated in apoptotic responses by small molecule chemotherapeutic agents. Next, we determined whether FC101-induced cell death involves MAPKs, namely activation of Erk1/2 and p38 downstream pathways. For this, HeLa cells were exposed to FC101 $(0-1 \mu \mathrm{M})$ for 48 hours, followed by immunoblot analysis. As shown in Figure 7, FC101 did not affect phosphorylation of ERK1/2, but induced phosphorylation of p38 in a concentrationdependent manner. Note: p-ERK1/2 level is often correlated to increased cell survival, whereas phosphorylated p38 level is frequently associated with stress response or cell death. This could suggest that FC101 induction of cancer cell death is in part due to the activation of the p38 MAPK signaling pathway.

The mTOR signaling pathway also plays a central role in the regulation of cell proliferation and growth [16].

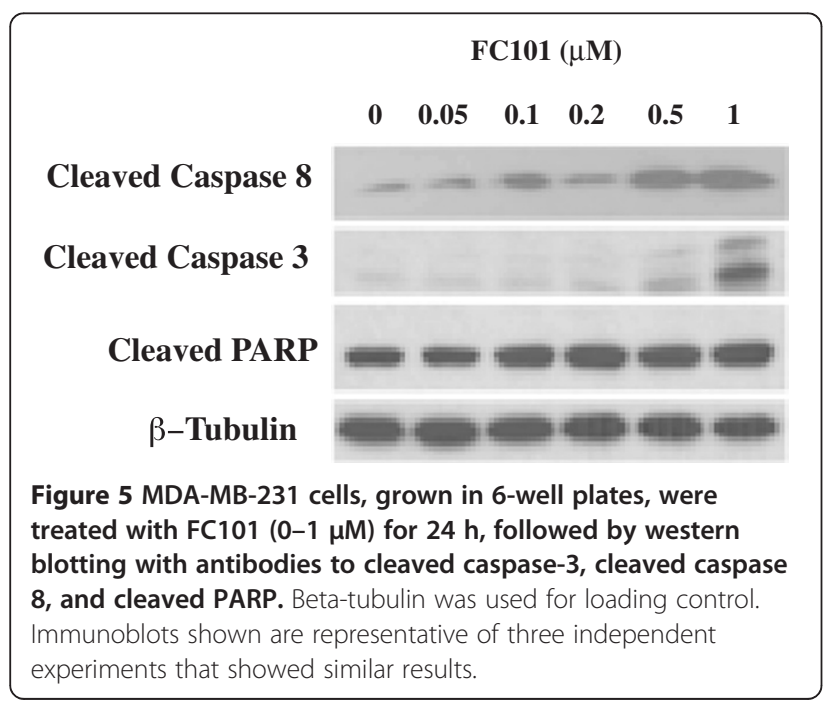

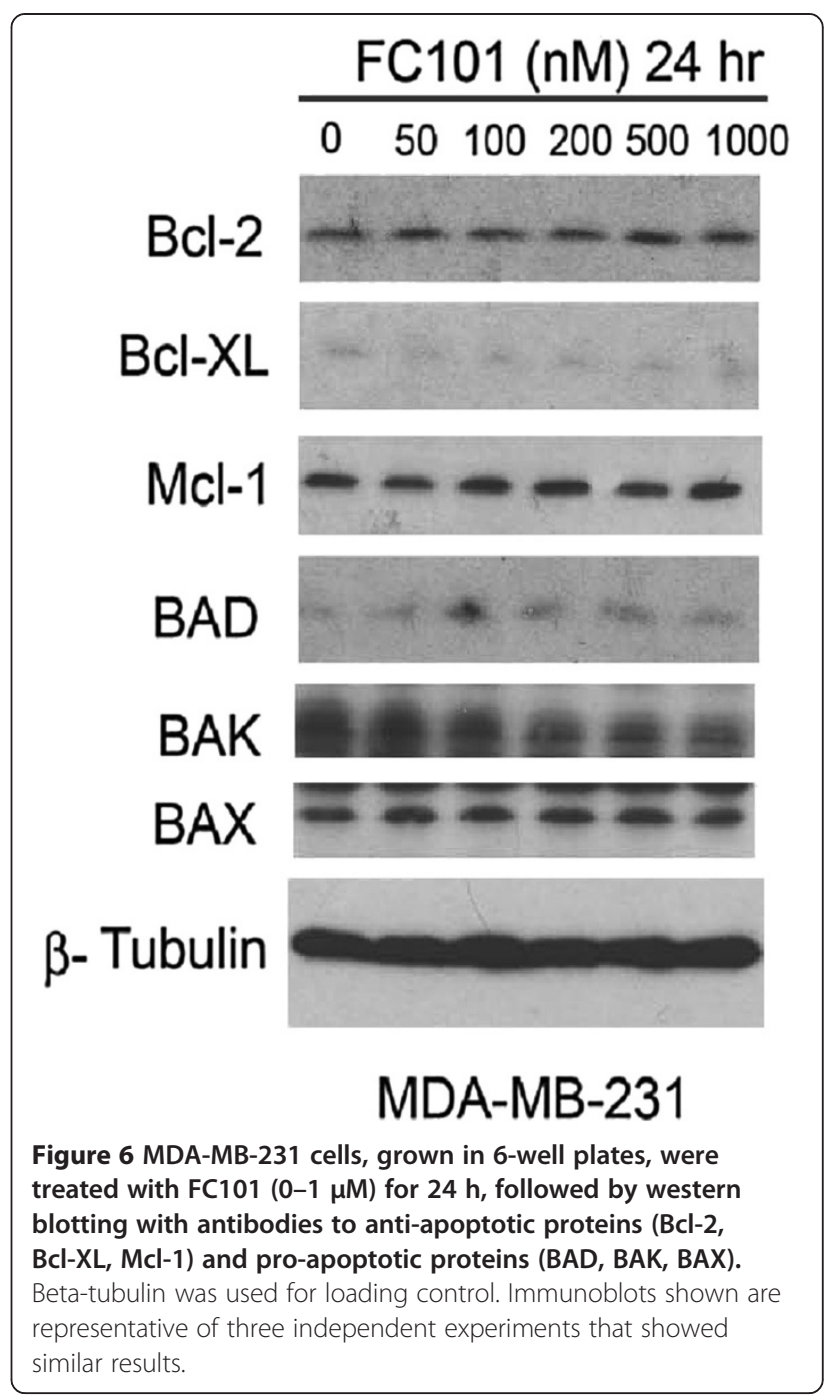

Inhibition of mTOR by rapamycin downregulates cyclin D1 expression [17] and Rb phosphorylation [18], resulting in cell cycle arrest during the $G_{1}-G_{0}$ phase. Since we found that FC101 arrests cells in $G_{1}-G_{0}$ phase of the cell cycle, we hypothesized that the cell-cycle modulatory effect of FC101 is linked to inhibition of the mTOR pathway. To test this hypothesis, we examined the effects of FC101 on mTOR signaling in Hela cells. As shown in Figure 7, FC101 lowers the levels of p-4E-BP1 (T37/46), one of the best-characterized downstream effector molecules of mTOR [16] in a concentrationdependent manner.

It is clear that the anti-proliferative and pro-apoptotic effects of FC101 are mediated through both the activation of p38-MAPK and the inhibition of mTOR signaling pathways. Further investigation of the underlying molecular mechanisms may lead to the design of other novel small molecules targeting both the mTOR and MAPK pathways. 


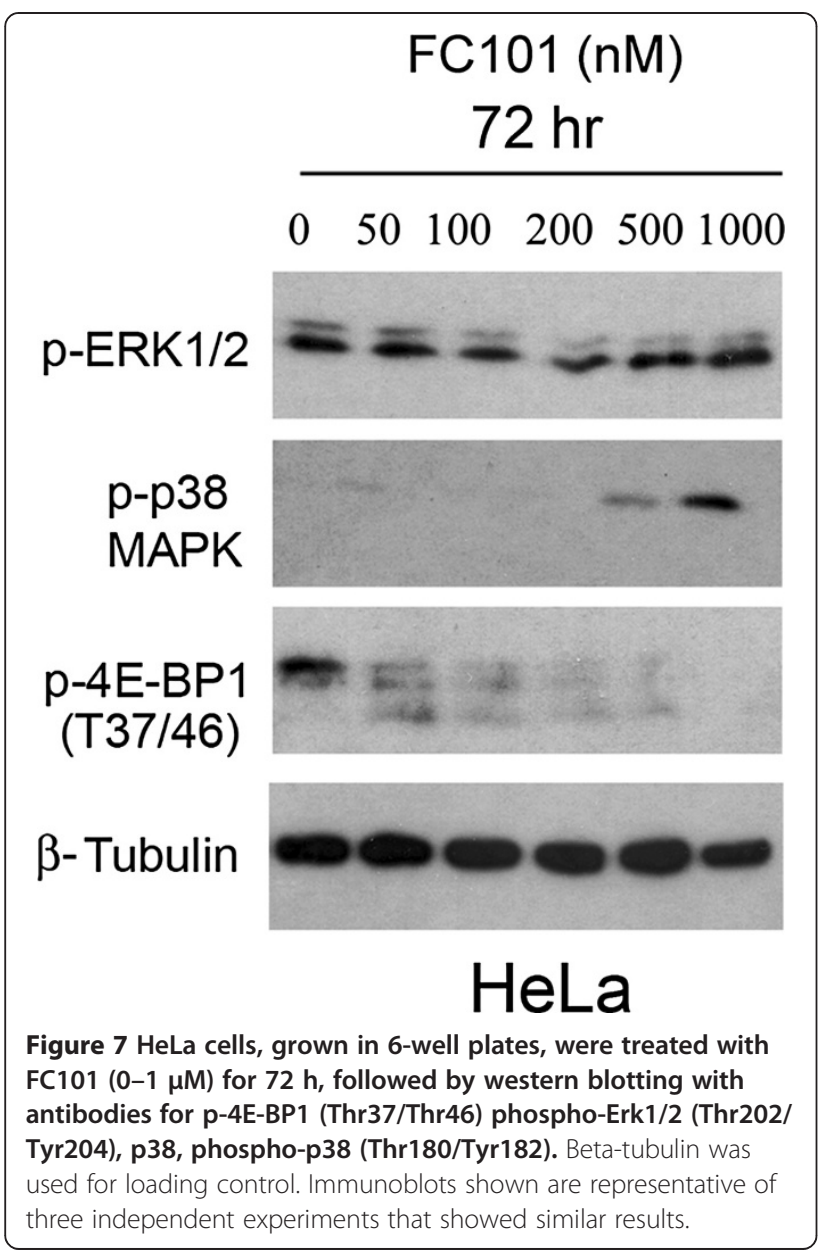

We also investigated the effects of FC101 on multidrug resistant (MDR) cells, MCF-7/Dox. We found that FC101 has a more pronounced anti-cancer effect ( 8fold lower $\mathrm{IC}_{50}$ ) on MCF-7/Dox than on their parental cell line, MCF-7 (Figure 8). The MCF-7/Dox cells overexpress two major genes responsible for drug resistance, one of which is glycosylceramide synthase (GCS) [19]. The fact that FC101 displays greater potency towards MDR cells suggests that it should be further explored as a sensitizing agent that may offer a therapeutic advantage. We are also currently designing experiments to more fully elucidate the detailed mechanism underlying this favorable feature of FC101.

Like most other bioactive natural flavonoids, FC101 has modest in-vivo activity. In a mouse xenograft skin SCC tumor model, FC101 was well tolerated and non-toxic, but it required a dose of $8 \mathrm{mg} / \mathrm{kg} /$ day treatment to achieve a $30 \%$ reduction in tumor size, compared to untreated controls (Figure 9, compare pink line with blue line).

To assess the direct anti-angiogenic properties of FC101, we used the MS1 mouse microvascular endothelial cell line, which was selected for its high VEGFR2 expression and responsiveness to VEGF functions [20].

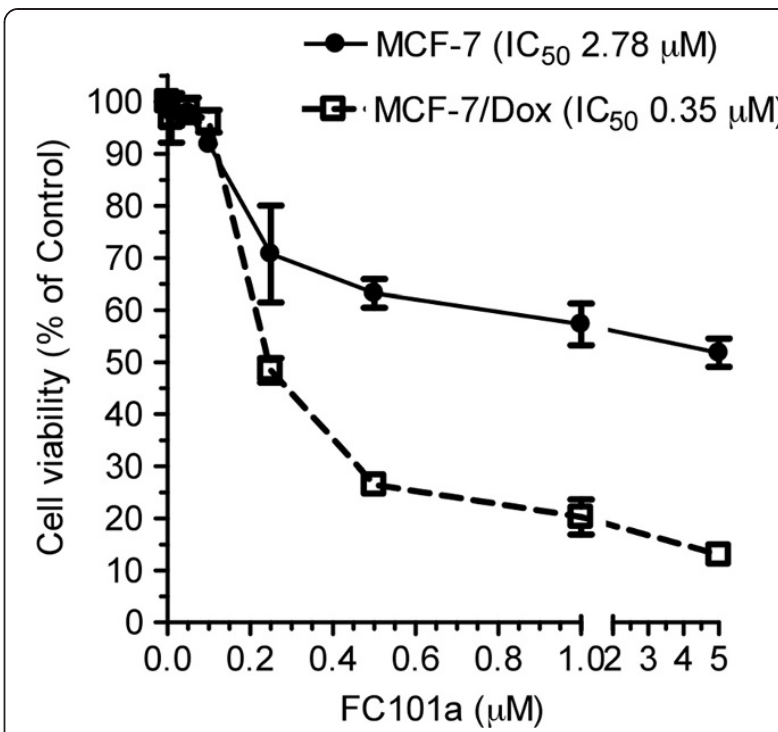

Figure 8 The growth inhibitory effect of FC101 on the MCF-7 and MDR MCF-7/Dox cells.

Thus, these cells are a good in-vitro proxy for optimal VEGF dependent responses that would be seen in vivo. FC101 displayed potent growth inhibition of MS1 cells with an $\mathrm{IC}_{50}<50 \mathrm{nM}$ after 4 hours treatment. The dosedependent effect of FC101 on VEGF-stimulated MS1 cell growth was also examined using an ELISA-based BrdU incorporation assay. We determined that FC101 significantly inhibits VEGF-dependent endothelial cell proliferation at all doses beginning at $10 \mathrm{nM}$ (Figure 10).

\section{Discussion}

FC101 is a small molecule fungal metabolite that has very potent anti-angiogenic activity and direct anticancer activity, and it exhibits potent in-vitro growth inhibitory effects (IC50 $<2.5 \mu \mathrm{M}$ for all cell lines tested). However, the precise biological target of FC101 remains unknown.

The data show that FC101 has the potential to be an effective anti-cancer therapeutic drug, because it suppresses both angiogenesis and tumorigenesis. In-vitro and in-vivo observations both indicate that FC101 is a powerful cytotoxic agent with strong selectivity towards cancer cells versus normal cells. Its broad-spectrum inhibitory effect on cancer cell lines and its direct inhibition of endothelial cell growth suggest that it could be beneficial for treating a variety of human cancers. In-vitro experiments showed significant differential sensitivity in a variety of malignant cell types (e.g., melanoma) vs. pig-1 melanocytes, immortalized non-malignant skin cells that serve as a control for human melanoma cell lines ${ }^{8}$. Cellular proliferation was measured by $3 \mathrm{H}$-thymidine incorporation (cpm/well). FC101 inhibited greater than $90 \%$ of incorporation with as little as $10 \mathrm{nM}$ in melanoma cells. In contrast, normal 


\section{FC101a, $8 \mathrm{mg} / \mathrm{kg} /$ day IP injection}

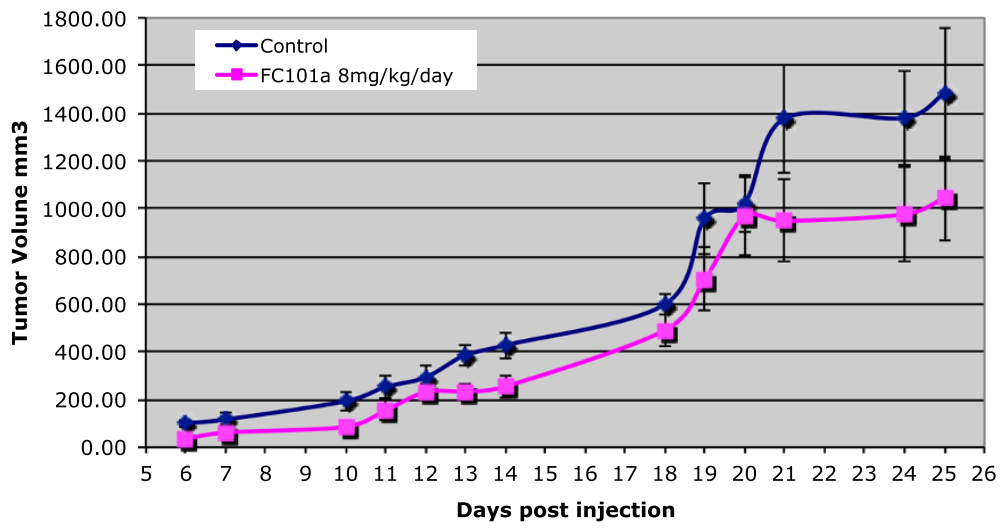

Figure 9 Immunocompromised mice (SCID) were injected subcutaneously with $1 \times 10^{6}$ SRB12-p9 cells on day 0 , and injected intraperitoneally with $8 \mathrm{mg} / \mathrm{kg} /$ day FC101 dissolved in PBS, or PBS alone as a control. The tumor volumes were determined by caliper measurement on the days indicated.

melanocytes were significantly less sensitive to FC101 (ca. 100-fold; data are not shown). In fact, FC101 dosages of 0.1 and $1 \mathrm{ng} / \mathrm{mL}$ caused stimulation of melanocyte proliferation; inhibition occurred only after exposure to high levels of FC101 for prolonged period of time, 8-12 days. An in vivo mouse xenograft SCC tumor model has also shown that FC101 is well tolerated and non-toxic to normal animal tissues.

Another unique attribute of FC101 was its differential growth inhibitory effect towards chemotherapy-resistant cancer cells. FC101 displayed a very potent sub-micromolar $\mathrm{IC}_{50}$ value against the MDR cells, MCF-7/Dox, which overexpress the enzyme, GCS. GCS converts ceramide to glucosylceramide, thereby deactivating it. Ceramide is an important lipid second messenger that mediates growth arrest and apoptosis of cells. Ceramide-induced apoptosis contributes to the therapeutic efficiencies of anthracyclines, taxanes, cytokines, and irradiation, and the overexpression of GCS has been identified as a possible

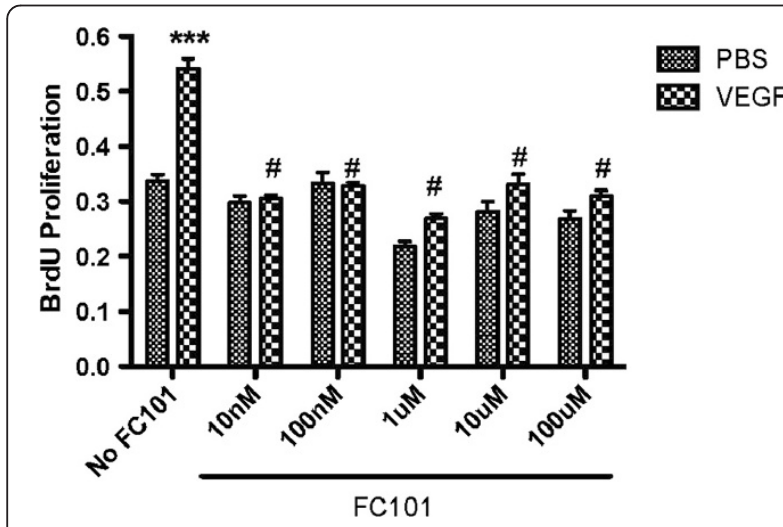

Figure 10 Suppression of VEGF-induced proliferation of mouse microvascular endothelial cells (MS1). mechanism for resistance to chemotherapy in a number of human cancers [19]. Indeed, GCS overexpression is displayed in MDR cell lines of breast, ovarian, cervical, and colorectal cancers [16]. Furthermore, these same investigators have demonstrated that inhibition of GCS sensitizes MDR cells to anticancer drugs [19,21]. Inhibition of GCS with small molecules, such as D-threo-1-phenyl-2decanoylamino-3-morpholino-1-propanol (PDMP), sensitizes cancer cells to doxorubicin, paclitaxel and vincristine [22]. The suppression of GCS expression by MBO-asGCS restores ceramide signaling (particular $\mathrm{C}_{18}$-ceramide) during the course of doxorubicin treatment, thereby promoting caspase-executed apoptotic death in cells. Thus, targeting GCS would promote caspase-executed apoptotic signaling mediated by ceramide, thereby eliminating tumors with poor response to conventional chemotherapy, such as doxorubicin. However, a therapeutic agent that efficiently inhibits GCS in vivo remains to be developed. FC101 is a promising candidate for such an agent. Our future studies will address whether FC101 suppresses GCS expression or activity to reverses drug resistance.

Figure 4 illustrates that FC101 has at least one other beneficial biological effect besides apoptosis induction. The increased fraction of cells in the $2 \mathrm{~N}$ DNA content peak G2 and M phases of the cell cycle suggests that FC101 could slow the rate of cell proliferation, possibly through an effect on the transition from G2 to mitosis. Further experiments, such as detailed analysis of cell cycle protein levels and phosphorylation states as well as cytological observation of treated cells using DNA staining, will be necessary to distinguish between cells in G2 and M phase.

We have shown that FC101's molecular mode of action involves three key cellular mechanisms including, extrinsic apoptosis, MAPK, and mTOR signaling pathways. FC101 
activates caspase-3, which initiates degradation of the cell in the final stages of apoptosis. Increased PARP cleavage confirms the activation of the caspase cascade. FC101 also activates caspase-8, as evidenced by increased caspase- 8 cleavage, further substantiating that FC101 induces apoptosis through an extrinsic pathway.

FC101 does not affect the expression of the antiapoptotic proteins, $\mathrm{Bcl}-2$, Bcl-XL, $\mathrm{Mcl}-1$, or the proapoptotic proteins BAD, BAK, BAX. This indicates that FC101's induction of apoptosis does not occur through an intrinsic mechanism. The data support the notion that FC101 induces caspase-dependent apoptosis, which implies that the extrinsic pathway is involved in this process. Future experiments will explore the effects of FC101 on the expression of death-receptor signaling proteins (TNF- $\alpha$ and FAS) to further confirm that FC101-induction of apoptosis is death receptor mediated through an extrinsic pathway.

FC101 did not affect phosphrorylation of ERK1/2, but it induced phosphorylation of p38 MAPK in a concentration-dependent manner (HeLa cells, $0-1 \mu \mathrm{M}$ ). Note p-ERK1/2 levels are often correlated to increased cell survival, whereas p38 levels are frequently associated with stress response or cell death. In addition, FC101 inhibits the phosphorylation of 4E-BP1 (T37/46), a substrate of mTOR. Since mTOR is a master kinase that controls cell growth and survival, this suggests that FC101 may induce cell death by simultaneously activating p38 MAPK and inhibiting mTOR signaling.

We have found that the cell-permeable small molecule, FC101, affects a diverse group of biological targets involved in cellular signal transduction. Collectively, our findings suggest that FC101 induces a series of caspasedependent events in the extrinsic cascade, resulting in apoptosis, and we have shown that FC101 simultaneously affects two downstream protein substrates involved in two distinct kinase signaling pathways (mTOR and MAPK signaling pathways).

We believe that FC101 holds promise as an anticancer agent due to its unique molecular mechanism especially as a modulator of both mTOR and MAPK, two master kinases that regulate cell proliferation and growth [16]. Further elucidation of the underlying molecular mechanism may lead to the design of a new class of targeted cancer therapeutics.

Our data also shows that FC101 inhibits VEGFdependent proliferation of microvascular endothelial cells (MS1 cells), indicating a strong anti-angiogenic effect. As shown in Figure 10, all doses of FC101 significantly inhibited VEGF-mediated Brdu incorporation. One limitation of prior work is that it was performed in the absence of any endothelial cell growth factors. This made it unclear how effective FC101 would be in conjunction with a growth factor like VEGF.
FC101 has shown high potency in vitro, coupled with low toxicity toward normal tissues and little or no adverse effects in various animal studies. In one report on the use of FC101 in a melanoma xenograft model in mice, Dréau et al. commented on the potent cytotoxicity of FC101, but also noted only minimal anti-angiogenic activity, despite the strong activation of caspase-3 at the periphery [12]. This model also confirmed other previous reports that $\mathrm{FC} 101$ is less potent in-vivo than in-vitro. Identifying the pharmacokinetic parameters of biologically active compounds is an essential step in the development of potential drug candidates. Typical pharmacokinetics parameters are the in vivo absorption, distribution, metabolism, and elimination of compounds in blood and tissues over time. FC101 is a flavonoid, and over 4,000 natural compounds have been characterized as such. These molecules are usually poorly to moderately water-soluble. They are also rapidly metabolized and degraded in vivo. Flavonoids generally bind tightly to serum proteins (e.g., serum albumin) and thus substantial amounts are inaccessible to the desired biological targets.

Through a previous study in Dr. Wuthier's lab (one of our collaborators), we have seen that FC101 does indeed bind to BSA with a binding constant of $2.47 \pm 0.17 \times 10^{8}$. To achieve efficacy in vivo, promising preclinical drug candidates like FC101 need to exploit pharmacologic delivery routes that facilitate sustained release of the active form of the drug at the desired site of action. Incorporation of flavonoids into lipid- or polymer-based nanoparticles appears promising. This markedly helps drug delivery, as these nanoparticles can protect the drug from degradation in the gastrointestinal tract and degradation from first-pass metabolism in the liver, by virtue of the unique absorption mechanism of these conjugates through the lymphatic system.

Thus, although we view FC101 as a promising drug candidate for structure-based lead optimization, its stability, bioavailability, and in vivo distribution appear to be sub-optimal. Currently, we are synthesizing new FC101 analogs with greater in-vivo potency and also attempting to identify the precise biological target(s) of FC101.

\section{Conclusion}

Scientists have traditionally turned to nature to find new lead compounds for fighting disease. Fungus and molds have produced many important new lead compounds for drug development. That is how FC101 was discovered, and it represents an important new lead compound for the treatment of cancer. However, the precise biological targets of FC101 remain unknown. Thanks to advances in computer-based assays and chemical synthesis, there are new ways to predict a drug's mechanism of action 
and to synthesize more biologically active analogs of promising lead compounds. The chemical synthesis of novel analogs, cell-based as well as protein-based assays that probe specific signaling pathways will play a significant role in our future development of FC101 for cancer therapy.

\section{Methods}

\section{Source of drug}

FC101 in its free base form (MW 292.3) was isolated and purified from Fusarium equiseti (originally supplied by Dr. Xie at the University of Minnesota). This fungus is found exclusively in the arctic latitudes and grown on rice cultures by Dr. Brian Furmanski. FC101 was then crystallized in the presence of phosphoric acid to form the more stable phosphate salt form (MW 390.3). Its purity (>98\%) was confirmed by ${ }^{1} \mathrm{H}-\mathrm{NMR},{ }^{13} \mathrm{C}-\mathrm{NMR}$, and UV-vis spectroscopy.

\section{Cell lines and culture}

Seven human tumor cell lines were used in this study. HaCat (pre-malignant skin, a gift from Prof. N.E. Fusenig, Deutsches Krebforschungszentrum), SRB12-p9 (malignant skin SCC, a gift from Dr. Reuben Lotan, Univ. of TexasMD Anderson Cancer Center), MCF-7 (low malignant mammary gland adenocarcinoma), MDA-231 (malignant mammary gland adenocarcinoma), SV-HUC (premalignant bladder), UM-UC14 (malignant bladder, a gift from Dr. H.B. Grossman, Univ. of Texas-MD Anderson Cancer Center), and PC3 (malignant, prostate). MCF-7, MDA231, SV-HUC and PC3 cells were obtained from the American Type Culture Collection (Manassas, VA). $\mathrm{HaCaT}$ cells were cultured in $4 \mathrm{x}$ modified Eagle's medium (MEM) media (1.4 $\left.\mathrm{mM} \mathrm{Ca}^{2+}\right)$, supplemented with 5\% fetal calf serum (FCS) and 1\% Penicillin Streptomycin Solution (Pen-Strep). SRB12-p9, MCF-7, MDA-MB231, and PC3 cells were cultured in Dulbecco's MEM (DMEM)/F12 medium containing 10\% FCS and 1\% Pen-Strep. UMUC14 cells were cultured in 50\% DMEM/F12 media and $50 \%$ DMEM low glucose media containing 10\% FCS and $1 \%$ Pen-Strep. SV-HUC cells were cultured in HyQ Ham's/F-12 media with 7\% FCS and 1\% Pen-Strep. For passaging, subconfluent cells were incubated with $0.1 \%$ trypsin/2 mM EDTA and suspended in media before replating. All cells were cultured at $37^{\circ} \mathrm{C}$ in the humidified atmosphere of $5 \% \mathrm{CO}_{2} / 95 \%$ air.

\section{MTT assays}

Cell viability was determined by the MTT 3-(4, 5dimethyltiazol 2-yl)-2,5-diphenyltetraolium bromide assay. Briefly, cells were plated in triplicate wells at varying densities (1,000 cells/well for HaCaT, SRB12-p9, MCF7, MDA-MB231; 1,500 cells/well for MCF7, PC3; 2,000 cells/well for SV-HUC) in $100 \mu$ l growth media in 96-well plates and treated with the FC101 at the concentrations indicated in Figure 4. After a range of treatment times a solution of MTT (20 $\mu$ l of a $12 \mathrm{mM}$ solution in PBS) was added and incubated for 2 hours at $37^{\circ} \mathrm{C}$. The cells were washed gently with PBS, and $100 \mu \mathrm{l}$ of DMSO was then added to the wells followed by mild shaking to dissolve the MTT precipitate. Absorbance at a wavelength of 540 $\mathrm{nm}$ was measured for each well using a Wallac Victor3 1420 Multilabel multi-well plate reader (Perkin-Elmer).

\section{Cell viability assays for multi-drug resistant MCF-7/Dox cells}

Cell viability was analyzed by quantitation of ATP, an indicator of active cells, using the CellTiter-Glo luminescent cell viability assay (Promega, Madison, WI). Briefly, cells $(4,000$ cells/well) were grown in 96-well plates with $10 \%$ FBS RPMI-1640 medium for $24 \mathrm{hr}$. FC101 was introduced into cells by Lipofectamine 2000 (vehicle control) in OptiMEM I reduced-serum medium, for a $4 \mathrm{hr}$ incubation. Cells were then incubated with increasing concentrations of FC101 in 5\% FBS medium for an additional $72 \mathrm{hr}$. Cell viability was determined by the measurement of luminescent ATP in a Synergy HT microplate reader, following incubation with CellTiter-Glo reagent. Triplicate experiments were repeated two times.

\section{Cell cycle analysis}

The cell cycle profile was determined by cell cycle flow cytometry based on cellular DNA content, using an Epics Profile II cell sorter (Coulter Electronics, Inc.). Cells were treated with FC101 or DMSO vehicle control for the indicated durations, trypsinized, collected and pelleted together with any material floating in the medium. Cells were fixed in cold 70\% ethanol, resuspended in PBS at a density of $>10^{6}$ cells $/ \mathrm{ml}$ followed by RNase A $(1 \mathrm{mg} / \mathrm{ml})$ treatment, addition of propidium iodide $(20 \mu \mathrm{g} / \mathrm{ml}$ final concentration) and analysis by flow cytometry. The percentage of cells in different phases of the cell cycle were determined from the raw data using the ModFit V3.2.1 flow cytometry software. Apoptosis was assessed by determining the amount of sub-G1 sized particles.

\section{Western blot analysis}

Western blotting was carried out as described previously [14]. Briefly, control and treated cells $(0-10 \mu \mathrm{M})$ were washed twice with cold PBS and then lysed in the lysis buffer [50 mmol/L Tris, pH 7.2; $150 \mathrm{mmol} / \mathrm{L} \mathrm{NaCl} ; 1 \%$ sodium deoxycholate; $0.1 \%$ SDS; $1 \%$ Triton X-100; 10 $\mathrm{mM} \mathrm{NaF}, 1 \mathrm{mM} \mathrm{Na} \mathrm{VO}_{4}$; Protease inhibitor cocktail (1:1000 dilution, Sigma). Lysates were sonicated for 10 seconds and centrifuged at 13,000 X g for 10 minutes at $4^{\circ} \mathrm{C}$. Protein concentration was determined using the BCA assay (Protein Assay Kit, Pierce). Protein samples $(\sim 40 \mu \mathrm{g})$ were electrophoresed on $8-12 \%$ SDS-PAGE, 
transferred to PVDF membrane (Millipore, USA), and probed with respective primary and secondary antibodies. The following primary antibodies were used: p38, phospho-p38 (Thr180/Tyr182), PARP, Bcl-2, Mcl-1, surviving, (all from Santa Cruz Biotechnology), Bcl-xL, BAK, BAX (Biomedia), p-4E-BP1(Thr37/Thr46), caspase 3 , cleaved caspase 3, cleaved caspase 8, BAD, phosphoErk1/2 (Thr202/Tyr204; Cell Signaling), and $\beta$-tubulin (Sigma). Goat anti-mouse Ig-G-horseradish peroxidase (HRP), goat anti-mouse IgM-HRP, and goat anti-rabbit IgG-HRP were purchased from Pierce.

\section{Anti-angiogenesis assay}

For our anti-angiogenic assays we used the murine MS1 microvascular endothelial cell line, selected due to its high VEGFR2 expression and responsiveness to VEGF. Inhibition of MS1 cell proliferation in the presence and absence of VEGF was evaluated using DNA incorporation of BrdU. MS-1 cells were serum starved overnight and the next morning preincubated with various concentrations of FC101 for 30 minutes. Next, $50 \mathrm{ng} / \mathrm{ml}$ VEGF or PBS alone (i.e., control) was added to respective wells along with BrdU and incubated for 4 hours. Cells were then fixed and BrdU incorporation measured by ELISA.

\section{In vivo tumorigenicity assay}

All animal studies were carried out following the USDA, PHS, and NIH animal use and care guidelines. The animal protocol was approved by the Institutional Animal Care and Use Committee at the Louisiana State University Health Science Center in Shreveport (LSUHSC-S) (Protocol Number, P10-045). SCID Beige mice (CB17/Icr.Cg-Prkdc ${ }^{\text {sci- }}$ ${ }^{\mathrm{d}} \mathrm{Lyst}^{\mathrm{bg}} / \mathrm{Crl}$ ) were maintained on AIN76A semi-purified diets (Dyets, Bethlehem, PA). Mice were administered FC101 or PBS by IP injections 5 days per week. Total daily drug intake was $8 \mathrm{mg} / \mathrm{kg}$. On day 3 , groups of 8 mice (6-7 week old) were injected subcutaneously (s.c.) in the dorsal region with $1 \times 10^{6}$ SRB12-p9 cells in $0.1 \mathrm{ml}$ PBS. Tumors were measured five times weekly by digital caliper using the formula $\mathrm{V}=((\mathrm{W}+\mathrm{L}) / 4))^{3} \times 4 / 3 \pi$, where $\mathrm{L}=$ length and $\mathrm{W}=$ width. Tumors were harvested and bisected at 25 days postinjection, or at the time of attaining a volume of $2 \mathrm{~cm}^{3}$. Tumors were fixed in formalin for histological analysis.

The differences between the groups of mice in terms of tumor volume or persisting benign cysts was compared using the Mann-Whitney test and differences in latency to tumor formation and survival were calculated by the Log-rank test and Kaplan-Meier Cumulative Survival tests.

\section{Statistical analysis}

Statistical analyses were completed using ANOVA, followed by Fisher's protected least significant difference procedure. A p-value of $<0.05$ (2-tail) was considered statistically significant.

\section{Abbreviations}

FC101: Fusarochromanone; VEGF-A: Vascular endothelial growth factor-A.

\section{Competing interests}

The authors declare that they have no competing interests.

\section{Authors' contributions}

EM participated in the design of the in-vitro and in-vivo assays for the assessment of biological function of Fusarochromanone and initiated the drafting of the manuscript. PP assisted in the western blotting experiments. SA assisted in the design and interpretation of western blotting experiments. TWH prepared the rice culture of fungus to obtain Fusarochromanone from biological sources. BF provided samples of Fusarochromanone which he obtained through biological source. YJK carried out some of the MTT assays and participated in cell cycle and apoptosis determination experiments. YG carried out some of the MTT and western blotting experiments. MB carried out some of the MTT and western blotting experiments. YW carried out some of the MTT and western blotting experiments. KNB performed the cell viability assays for multi-drug resistant MCF-7/Dox cells. GKK performed the VEGF-based inhibition of MS-1 proliferation through BrdU incorporation measured by ELISA. QQ carried out some of the MTT and western blotting experiments. YYL participated in the design of the cell viability assays for multi-drug resistant MCF-7/Dox cells and helped to draft the manuscript. CGK conceived of the design and coordination of the anti-angiogenic experiments and helped to draft the manuscript. BAS purified the Fusarochromanone obtained from both chemical and biological sources and helped to draft the manuscript. SH conceived of the design and coordination of the western blotting experiments and helped to draft the manuscript. $J \mathrm{LC}$ conceived of the design and coordination of the cell-based and animal based anti-cancer experiments and helped to draft the manuscript. All authors read and approved the final manuscript.

\section{Acknowledgements}

The project described here was supported by National Center for Research Resources (award \# 5P20RR016456-11) and the National Institute of General Medical Sciences (award \# 8 P20 GM103424-11) from the National Institutes of Health.

"The content is solely the responsibility of the authors and does not necessarily represent the official views of the National Center For Research Resources or the National Institute of Health". This work was also supported in part by National Cancer Institute grants CA135274 and CA135274-S1. We thank Jennifer Roberts for MTT assays and Debra Chervenak for FACS anaIysis. FACS equipment was made available through the Research Core Facility at Louisiana State University Health Sciences Center in Shreveport, LA.

\section{Assurance statement}

"All research was conducted under the supervision of researchers (Elahe Mahdavian, Associate Professor, Dept. of Chemistry, LSUS; John Clifford, Associate Professor, Dept. of Biochemistry, LSUHSC-Shreveport, and Tara Williams-Hart, Professor, Dept. of Biology, LSUS) and followed state and federal regulatory guidance applicable to the human and ethical conduct of such research".

\section{Author details}

'Department of Chemistry and Physics, LSU-Shreveport, One University Place, Shreveport, LA 71115, USA. ²Department of Biological Science, LSU-Shreveport, Shreveport, USA. ${ }^{3}$ GlaxoSmithKline, Research Triangle, Park, NC, USA. ${ }^{4}$ Department of Biochemistry and Molecular Biology, LSUHSC-Shreveport, Shreveport, USA. ${ }^{5}$ Department of Basic Pharmaceutical Sciences, University of Louisiana-Monroe, Monroe, USA. ${ }^{6}$ Department of Pathology, LSUHSC-Shreveport, Shreveport, USA. ${ }^{7}$ Department of Biological Sciences, Tennessee State University, Nashville, USA. ${ }^{8}$ US Army Institute of Surgical Research, Fort Sam, Houston, TX 78234, USA.

Received: 27 October 2013 Accepted: 15 August 2014

Published: 3 September 2014

\section{References}

1. Lee YW, Mirocha CJ, Shroeder DJ, Walser MM: TDP-1, a toxic component causing tibial dyschondroplasia in broiler chickens, and trichothecenes from fusarium roseum 'Graminearum'. Appl Environ Microbiol 1985, 50:102. 
2. WU W, Nelson PE, Cook ME, Smalley EB: Fusarochromanone Production by Fusarium Isolates. Appl Environ Microbiol 1990, 56(10):2993.

3. Walser MM, Morris VC, Levander OA: Effect of dietary selenium on the development of fusarium-induced tibial dyschondroplasia in broiler chickens. Avian Diseases 1988, 32:84.

4. Miloslav R: Handbook of Foodborne Diseases of Biological Origin (CRC Series in Nutrition and Food). Boca Raton, Florida: CRC Press; 1983:473.

5. Pathre SV, Gleason WB, Lee Y-W, Mirocha CJ: The Structure of fusarochromanone: New mycotoxin from Fusarium roseum. Gramineurum. Can J Chem 1986, 64:1308.

6. Xie WP, Mirocha CJ, Pawlosky RJ, Wen YC, Xu XG: Biosynthesis of fusarochromanone and its monoacetyl derivative by fusarium equiseti. Appl Environ Microbiol 1989, 55:794.

7. Nie D: Investigation of calcification and vascularization of the epiphyseal growth plate cartilage using avian tibial dyschondroplasia and hypervitaminosis as models, (Chapter III). "Effects of fusarochromanone on endothelial cells: implication of deficient vascularization in the pathogenesis of avian tibial dyschondroplasia. In Ph.D. Thesis, Department of Chemistry and Biochemistry. Columbia: University of South Carolina; 1997:100.

8. Furmanski BD, Dréau D, Wuthier RE, Fuseler JW: Differential uptake and selective permeability of fusarochromanone (FC101), a novel membrane permeable anticancer naturally fluorescent compound in tumor and normal cells. Microsc Microanal 1999, 15:545.

9. Lipinski CA: Experimental and Computational approaches to estimate solubility and permeability in drug discovery and development settings. Adv Drug Delivery Rev 2001, 46:3.

10. Kramer SD: Absorption prediction from physicochemical parameters. Pharma Sci \& Tech Today 1999, 2(9):141.

11. Veber DF: Molecular properties that influence the oral bioavailbility of drug candidates. J Med Chem 2002, 45:2615.

12. Dreau D, Foster M, Hogg M, Culberson C, Nunes P, Wuthier RE: Inhibitory effects of fusarochromanone on melanoma growth. Anticancer Drugs 2007, 18:897.

13. Madeo F, Herker E, Maldener C: A caspase-related protease regulates apoptosis in yeast. Mol Cell 2002, 9:911.

14. Pradelli $L A$, Beeteau M, Ricci JE: Mitochondrial control of caspase dependent and -independent cell death. Cell Mol Life Sci 2010, 67:1589.

15. Liu L, Chen L, Chung J, Huang S: Rapamycin inhibits F-actin reorganization and phosphorylation of focal adhesion proteins. Oncogene 2008, 27:4998.

16. Guertin DA, Sabatini DM: Defining the role of mTOR in cancer. Cancer Cell 2007, 12:9.

17. Hashemolhosseini S, Nagamine Y, Morley SJ, Desrivieres S, Mercep L, Ferrari $S$ : Rapamycin inhibition of the $\mathrm{G} 1$ to $\mathrm{S}$ transition is mediated by effects on cyclin D1 mRNA and protein stability. J Biol Chem 1998, 273:14424.

18. Chen Y, Knudsen ES, Wang JY: The RB/p107/p130 phosphorylation pathway is not inhibited in rapamycin-induced G1-prolongation of NIH3T3 cells. Oncogene 1996, 13:1765.

19. Liu YY, Han TY, Giuliano AE, Cabot MC: Ceramide glycosylation potentiates cellular multidrug resistance. FASEB J 2001, 15:719.

20. Arbiser JL, Moses MA, Fernandez CA, Ghiso N, Cao Y, Klauber N, Frank D, Brownlee M, Flynn E, Parangi S, Byers HR, Folkman J: Oncogenic H-ras stimulates tumor angiogenesis by two distinct pathways. Proc Natl Acad Sci U S A 1997, 94(3):861.

21. Liu YY, Yu JY, Yin D, Patwardhan GA, Gupta V: A role for ceramide in driving cancer cell resistance to doxorubicin. FASEB J 2008, 22:2541.

22. Lavie Y, Cao H, Volner A, Lucci A, Han TY: Agents that reverse multidrug resistance, tamoxifen, verapamil, and cyclosporin $\mathrm{A}$, block glycosphingolipid metabolism by inhibiting ceramide glycosylation in human cancer cells. J Biol Chem 1997, 272:1682.

doi:10.1186/1756-0500-7-601

Cite this article as: Mahdavian et al:: Biological activities of

fusarochromanone: a potent anti-cancer agent. BMC Research Notes 2014 7:601.

\section{Submit your next manuscript to BioMed Central and take full advantage of:}

- Convenient online submission

- Thorough peer review

- No space constraints or color figure charges

- Immediate publication on acceptance

- Inclusion in PubMed, CAS, Scopus and Google Scholar

- Research which is freely available for redistribution

Submit your manuscript at www.biomedcentral.com/submit
C Biomed Central 